\title{
3.
}

\section{Vereinfachung des Beweises von Cauchy, dafs jede Gleichung nten Grades wenigstens eine Wurzel hat.}

(Von Herrn J. Su/smarn, Assistent im Königlichen Gewerbe-Institut zu Berlin.)

Cauchy beweiset in seinem "Cours d'analyse ch. $X . "$ diesen Satz-in der Weise, dafs er für $x$ den Ausdruck $u+v \sqrt{ }-1$ einführt und die Function $\boldsymbol{F}(\boldsymbol{u}, \boldsymbol{v})=[\varphi(\boldsymbol{u}, \boldsymbol{v})]^{2}+[\psi(\boldsymbol{u}, \boldsymbol{v})]^{2}$ aufstellt. Dann zeigt er auf eine sehr umständliche Art, dafs diese Function ein Minimum habe, und dafs dieses Minimum gleich Null sein müsse. Daraus folgt dann, dafs auch $f(u+v \sqrt{-1})=f(x)$ für denselben Werth von $u$ und $v$ verschwindet.

Wie es scheint, läfst es sich leichter zeigen, dafs die Function $\boldsymbol{F}(\boldsymbol{u}, \boldsymbol{v})$ ein Minimum habe, wenn man den Gang der Function beobachtet, während $u$ und $v$ sich stetig von $-\infty$ bis $+\infty$ ändern. Es ergiebt sich dann sehr einfach, dafs dieses Minimum gleich Null ist, wenn man ein bekanntes Gesetz aus der Differentialrechnung heranzieht.

Der Beweis gestaltet sich folgendermaafsen.

Es sei die geordnete Gleichung

$$
\boldsymbol{x}^{n}+\boldsymbol{a} \boldsymbol{x}^{n-1}+\boldsymbol{b} \boldsymbol{x}^{n-2}+\cdots+\boldsymbol{g} \boldsymbol{x}+\boldsymbol{k}=\mathbf{0}
$$

gegeben, wo $n$ eine positive ganze Zahl ist und $a, b, \ldots g, k$ beliebige reelle oder imaginäre bekannte Gröfsen sind.

Es werde $x$ veränderlich angenommen und der gegebene Ausdruck gleich $f(x)$ gesetzt. Für werde $u+v \sqrt{ }-1$ gesetzt; was, wenn man sich die Substitution ausgeführt vorstellt, einen Ausdruck von der Form

$$
\varphi(u, v)+\sqrt{ }-1 \psi(u, v)=f(x)
$$

giebt.

Man bilde noch die Function

$$
\boldsymbol{F}(\boldsymbol{u}, \boldsymbol{v})=[\varphi(\boldsymbol{u}, \boldsymbol{v})]^{2}+[\psi(\boldsymbol{u}, \boldsymbol{v})]^{2}
$$

Diese Function kann nie negativ werden. Setzt man nun $\boldsymbol{u}=-\infty$, während $v$ einen beliebigen endlichen Werth hat, so wird $\boldsymbol{F}(\boldsymbol{u}, \boldsymbol{v})=+\infty$. Denselben Werth erhält die Function für $u=+\infty$ und für einen beliebigen Werth von $v$. Eben so wird $F(u, v)=+\infty$ für beliebige endliche Werthe von $u$, wenn $v$

Crelle's Journal f. d. M. Bd. XLIV. Heft 1. 
58 3. Sufsmann, vom Satze, da/s jede Gl. wenigstens eine Wurzel hat.

gleich $+\infty$ oder $-\infty$ ist. Haben dagegen $u$ und $v$ gleichzeitig endliche Werthe, so hat auch $F(u, v)$, weil es eine ganze Function von $u$ und $v$ ist, einen endlichen Werth. Läfst man nun $u$ und $v$ gleichzeitig sich stetig von $-\infty$ bis $+\infty$ ändern, so wird $F(u, v)$, welche Werthe von $u$ und vach zusammengehören mögen, von $+\infty$ bis ins Endliche fallen, und dann wieder aus dem Endlichen bis zu $+\infty$ aufsteigen. Daraus, folgt, da/s nothwendiy die Function ein Minimum hat.

Setzt man

$$
u+v \sqrt{-1}=r(\cos y+\sqrt{-1} \sin y)
$$

uñd die sich hieraus ergebenden Werthe von $u$ und $v$ in $F(u, v)$, so erhält man eine Function von $r$ und $y$. Die Substitution wird ausgeführt, wenn man $x=r(\cos y+\sqrt{-1} \sin y)$ in $f(x)$ setzt. Es ergiebt sich:

$\left\{\begin{array}{l}\boldsymbol{r}^{n} \cos [n y] \\ +a r^{n-1} \cos [(n-1) y] \\ +b r^{n-2} \cos [(n-2) y] \\ \cdot \cdot \cdot \cdot \cdot \cdot \cdot \cdot \\ +g r \cos y \\ +k\end{array}\right\}+\sqrt{-1}\left\{\begin{array}{c}r^{n} \sin [n y] \\ +a r^{n-1} \sin [(n-1) y] \\ +b r^{n-2} \sin [(n-2) y] \\ \cdot \cdot \cdot \cdot \cdot \cdot \cdot \cdot \\ +g r \sin y\end{array}\right\}=f(x)=f(u+v \sqrt{ } \cdot 1)$.

Der Kürze wegen werde der Ausdruck in den ersten Klammern durch $\boldsymbol{A}$, der in den zweiten durch $\boldsymbol{B}$ bezeichnet; dann ist

$$
\begin{gathered}
\varphi(\boldsymbol{u}, \boldsymbol{v})=\boldsymbol{A} ; \quad \psi(\boldsymbol{u}, \boldsymbol{v})=\boldsymbol{B} \text { und } \\
\boldsymbol{F}(\boldsymbol{u}, \boldsymbol{v})=\boldsymbol{A}^{2}+\boldsymbol{B}^{2} .
\end{gathered}
$$

Es wurde nachgewiesen, dafs diese Function ein Minimum hat. Für dieses Minimum mufs, nach bekannten Gesetzen, die Ableitung von $\boldsymbol{A}^{2}+\boldsymbol{B}^{2}$, sowohl nach $y$ als nach $r$, gleich Null sein, wenn man in die Ableitung diejenigen Werthe von $\boldsymbol{r}$ und $y$ setzt, welche die Function zu einem Minimum machen.

Die Ableitung nach $y$ ist

$$
\left\{\begin{array}{l}
-\boldsymbol{A}\left(\boldsymbol{r}^{n} n \sin [n y]+a r^{n-1}(n-1) \sin [(n-1) y]+\cdots g r \sin y\right) \\
+\boldsymbol{B}\left(\boldsymbol{r}^{n} n \cos [n y]+a r^{n-1}(n-1) \cos [(n-1) y]+\cdots g r \cos y\right)
\end{array}\right\}=0
$$

oder, durch $r$ dividirt:

$$
\left\{\begin{array}{l}
-\boldsymbol{A}\left(n r^{n-1} \sin [n y]+a(n-1) r^{n-2} \sin [(n-1) y]+\cdots g \sin y\right) \\
+B\left(n r^{n-1} \cos [n y]+a(n-1) r^{n-2} \cos [(n-1) y]+\cdots g \cos y\right)
\end{array}\right\}=0 .
$$

Die Ableitung nach $r$ ist:

$$
\left\{\begin{array}{r}
\boldsymbol{A}\left(n r^{n-1} \cos [n y]+a(n-1) r^{n-2} \cos [(n-1) y]+\cdots g \cos y\right) \\
+\boldsymbol{B}\left(n r^{n-1} \sin [n y]+a(n-1) r^{n-2} \sin [(n-1) y]+\cdots g \sin y\right)
\end{array}\right\}=0 .
$$


5. Sufsmann, vom Satze, dafs jede Gl. wenigstens eine Wurzel hat. 59

Bezeichnet man der Kürze wegen den Factor von $A$ in (1.) und den von $B$ in (2.) durch $C$; ferner den Factor von $B$ in (1.) und den von $A$ in (2.) durch $\boldsymbol{D}$, so nehmen die Gleichungen (1.) und (2.) die Gestalt:

$$
\begin{aligned}
& \boldsymbol{A C}=+\boldsymbol{B D}, \\
& \boldsymbol{A D}=-\boldsymbol{B C}
\end{aligned}
$$

an. Multiplicirt man diese Gleichungen mit einander, so erhält man

$$
\boldsymbol{A}^{2}+\boldsymbol{B}^{2}=\mathbf{0}
$$

D. h. die Werthe von $u$ und $v$, welche die Function $F(u, v)$ zu einem Minimum machen, machen dieses Minimum zu Null. Es kann aber $\boldsymbol{F}(\boldsymbol{u}, v)$ nur dann zu Null werden, wenn gleichzeitig $\varphi(u, v)=0$ und $\psi(u, v)=0$ ist, also ist auch

folglich der Satz bewiesen.

$$
\varphi(u, v)+\sqrt{ }-1) \psi(u, v)=f(x)=0,
$$

Berlin, im Januar 1852. 